\title{
Multi-Innovation Stochastic Gradient Parameter and State Estimation Algorithm for Dual-Rate State-Space Systems with $d$-Step Time Delay
}

\author{
Ya Gu (D, ${ }^{1}$ Quanmin Zhu ${ }^{(D},{ }^{2}$ Jicheng Liu, ${ }^{1}$ Peiyi Zhu, ${ }^{1}$ and Yongxin Chou ${ }^{1}{ }^{1}$ \\ ${ }^{1}$ School of Electrical Engineering and Automation, Changshu Institute of Technology, Changshu 215500, Jiangsu, China \\ ${ }^{2}$ Department of Engineering Design and Mathematics, University of the West of England, Bristol BS16 1QY, UK \\ Correspondence should be addressed to Ya Gu; guya927819@163.com
}

Received 22 May 2020; Revised 16 June 2020; Accepted 22 June 2020; Published 13 July 2020

Guest Editor: Qiang Chen

Copyright (c) $2020 \mathrm{Ya} \mathrm{Gu}$ et al. This is an open access article distributed under the Creative Commons Attribution License, which permits unrestricted use, distribution, and reproduction in any medium, provided the original work is properly cited.

\begin{abstract}
This paper presents a multi-innovation stochastic gradient parameter estimation algorithm for dual-rate sampled state-space systems with $d$-step time delay by the multi-innovation identification theory. Considering the stochastic disturbance in industrial process and using the gradient search, a multi-innovation stochastic gradient algorithm is proposed through expanding the scalar innovation into an innovation vector in order to obtain more accurate parameter estimates. The difficulty of identification is that the information vector in the identification model contains the unknown states. The proposed algorithm uses the state estimates of the observer instead of the state variables to realize the parameter estimation. The simulation results indicate that the proposed algorithm works well.
\end{abstract}

\section{Introduction}

The mathematical model can represent the basic features of the system, and system identification applies the statistical methods to set up the mathematical models of dynamic systems from available data [1-4]. There exist some identification methods for state-space models with and without state-delay $[5,6]$, such as the recursive least squares (RLS) algorithm and the stochastic gradient (SG) algorithm. The SG algorithm is used in adaptive control because of its small computation. Recently, Chen et al. presented an Aitken based the modified Kalman filtering stochastic gradient algorithm for dual-rate nonlinear models [7]. Many identification methods have been developed for linear stochastic systems [8-11], bilinear stochastic systems [12-14], and nonlinear systems with colored noises [15-17].

In system identification, some algorithms concentrate on reducing the calculation amount and improving the recognition accuracy [18-20]. Since the gradient optimization method only requires calculating the first-order derivative, its computation is small [21]. However, the calculation accuracy of the gradient algorithm is low [22]. In order to improve the calculation accuracy, many improved gradient algorithms have been proposed. Although these improved algorithms can improve the parameter estimation accuracy, the computational complexity is large. The innovation is effective information to improve the parameter estimation accuracy $[23,24]$. It can promote the convergence of the algorithm in the recursive process. In order to improve the estimation accuracy through using more innovation, the multi-innovation theory has been used in system recognition.

The stabilities and identification of time-delay systems have drawn a great deal of attention of many researchers in system control and system analysis [25]. In industrial processes and control systems, time delays are difficult to avoid due to material transmission and signal interruptions. The time delay makes it difficult for the control system to respond to the input changes in time [26]. In addition, the time delay can cause instability and unsatisfactory 
performance of the controlled process. The recognition of time-delay systems has been a hot topic [27]. For example, Sanz et al. studied an observation and stabilization of LTV systems with time-varying measurement delay [28]. Li et al. discussed the local discontinuous Galerkin method for reaction-diffusion dynamical systems with time delays [29].

This paper studies identification problems of a dual-rate state-space model with $d$-step time delay. The main contributions of this paper are as follows. The input-output representation is derived from a canonical state-space model of the state-delay system for the identification through eliminating the state variables in the systems, to derive a joint parameter and state estimation algorithm by means of the multi-innovation identification theory and the state observer for reducing the computational burden and improving the parameter estimation accuracy and the convergence speed.

This paper is organized as follows: Section 2 gives the canonical state-space model for state-delay systems; Section 3 introduces the identification model; Section 4 presents a combined multi-innovation stochastic gradient (MISG) parameter and state estimation algorithm; Section 5 provides an illustrative example; and finally, we offer some concluding remarks in Section 6.

\section{The Canonical State-Space Model for State- Delay Systems}

Let us introduce some symbols. The relation $A:=X$ or $X:=A$ means that $A$ is defined as $X ; \mathbf{I}\left(\mathbf{I}_{n}\right)$ stands for an identity matrix of appropriate size $(n \times n) ; z$ denotes a unit forward shift operator: $z x(k)=x(k+1)$; $T$ represents the matrix/ vector transpose; $\hat{\theta}(k)$ is the estimate of $\theta$ at time $k ; \mathbf{1}_{n}$ means that an $n \times 1$ vector whose elements are all unity; $E$ denotes the expectation operator; $\operatorname{adj}[\mathbf{X}]$ stands for the adjoint matrix of the square matrix $\mathbf{X}: \operatorname{adj}[\mathbf{X}]=\operatorname{det}[\mathbf{X}] X^{-1} ; \operatorname{det}[\mathbf{X}]=|\mathbf{X}|$ represents the determinant of the square matrix $\mathbf{X}$.

Consider the following state-space system with $d$-step state-delay:

$$
\begin{aligned}
\mathbf{x}(k+1) & =\mathbf{A} \mathbf{x}(k)+\mathbf{B} \mathbf{x}(k-d)+\mathbf{f} u(k), \\
y(k) & =\mathbf{c x}(k)+w(k), \\
w(k) & =\sum_{r=1}^{n_{g}} g_{r} v(k-r)+v(k),
\end{aligned}
$$

$$
\begin{aligned}
\mathbf{A} & :=\left[\begin{array}{ccccc}
0 & 1 & 0 & \cdots & 0 \\
0 & 0 & 1 & \ddots & \vdots \\
\vdots & \vdots & \ddots & \ddots & 0 \\
0 & 0 & \cdots & 0 & 1 \\
a_{n} & a_{n-1} & a_{n-2} & \cdots & a_{1}
\end{array}\right] \in \mathbb{R}^{n \times n}, \\
\mathbf{B} & :=\left[\begin{array}{c}
b_{1} \\
b_{2} \\
\vdots \\
b_{n}
\end{array}\right] \in \mathbb{R}^{n \times n}, \\
\mathbf{b} & \in \mathbb{R}^{1 \times n}, \\
\mathbf{f} & :=\left[f_{1}, f_{2}, \ldots, f_{n}\right]^{T} \in \mathbb{R}^{n} \\
\mathbf{c} & :=[1,0,0, \ldots, 0] \in \mathbb{R}^{1 \times n},
\end{aligned}
$$

where $x(k) \in \mathbb{R}^{n}$ is the system state vector, $u(k) \in \mathbb{R}$ is the system input, $y(k) \in \mathbb{R}$ is the system output, $v(k) \in \mathbb{R}$ is a random noise with zero mean, and $\mathbf{A} \in \mathbb{R}^{n \times n}, \mathbf{B} \in \mathbb{R}^{n \times n}$, $\mathbf{f} \in \mathbb{R}^{n}$, and $\mathbf{c} \in \mathbb{R}^{1 \times n}$ are the system parameter matrices/ vectors. Assume that (c, $\mathbf{A})$ is observable and $u(k)=0$ and $y(k)=0$ for $k \leq 0$. The system matrices/vectors $\mathbf{A}, \mathbf{B}$, and $\mathbf{f}$ are the unknown parameters to be estimated from the inputoutput data $\{u(k), y(k)\}$. If we remove $\mathbf{B x}(k-d)$ in equation (1), then it becomes the conventional standard state-space model.

Remark 1. For the system in (1) and (2), if the state vector $x(k)$ is known, the system matrix/vector $(\mathbf{A}, \mathbf{b})$ is easy to identify. This paper considers the case that the state $x(k)$ is completely unavailable. The objective is to propose new methods for jointly estimating the unknown states and parameters from the measurement data $\{u(k), y(k): k=1,2, \ldots\}$ and to study the performance of the proposed methods.

\section{The Identification Model}

This section derives the identification model of the canonical state-space model in (1) and (2). From (1), we have

$$
\begin{gathered}
x_{i}(k+1)=x_{i+1}(k)+b_{i} x(k-d)+f_{i} u(k), \\
i=1,2, \ldots, n-1, \\
\begin{aligned}
x_{n}(k+1)= & a_{n} x_{1}(k)+a_{n-1} x_{2}(k)+\cdots+a_{1} x_{n}(k) \\
& +b_{n} x(k-d)+f_{n} u(k) .
\end{aligned}
\end{gathered}
$$


Let $\mathbf{a}:=\left[a_{n}, a_{n-1}, \ldots, a_{1}\right]^{T} \in \mathbb{R}^{n}$, using the properties of the shift operator $z$, multiplying (5) by $z^{-i}$ and (6) by $z^{-n}$, and adding all expressions give

$$
\begin{aligned}
x_{1}(k)= & \mathbf{a x}(k-n)+\mathbf{b}_{1} \mathbf{x}(k-d-1)+\mathbf{b}_{2} \mathbf{x}(k-d-2)+\cdots+\mathbf{b}_{n-1} \mathbf{x}(k-d-n+1) \\
& +\mathbf{b}_{n} \mathbf{x}(k-d-n)+f_{1} u(k-1)+f_{2} u(k-2)+\cdots+f_{n} u(k-n) \\
= & \mathbf{a x}(k-n)+\sum_{i=1}^{n} \mathbf{b}_{i} \mathbf{x}(k-d-i)+\sum_{i=1}^{n} f_{i} u(k-i) .
\end{aligned}
$$

When $d \leq n-1$, define the information vector $\varphi(k)$ and the parameter vector $\theta$ :

$$
\begin{aligned}
\boldsymbol{\varphi}(k) & :=\left[\boldsymbol{\varphi}_{1}^{\mathrm{T}}(k), \boldsymbol{\varphi}_{n}^{\mathrm{T}}(k)\right]^{\mathrm{T}}, \\
\boldsymbol{\varphi}_{1}(k) & :=\left[\begin{array}{r}
\left.\mathbf{x}^{\mathrm{T}}(k-d-1), \mathbf{x}^{\mathrm{T}}(k-d-2), \ldots, \mathbf{x}^{\mathrm{T}}(k-n), \ldots, \mathbf{x}^{\mathrm{T}}(k-n-d),\right]^{\mathrm{T}} \in \mathbb{R}^{n^{2}+n}, \\
u(k-1), u(k-2), \ldots, u(k-n)
\end{array}\right] \\
\boldsymbol{\varphi}_{n}(k) & :=\left[v(k-1), v(k-2), \ldots, v\left(k-n_{g}\right)\right]^{\mathrm{T}} \in \mathbb{R}^{n_{g}}, \\
\boldsymbol{\theta} & :=\left[\boldsymbol{\theta}_{1}^{\mathrm{T}}, \boldsymbol{\theta}_{n}^{\mathrm{T}}\right]^{\mathrm{T}}, \\
\boldsymbol{\theta}_{1} & :=\left[\mathbf{b}_{1}, \mathbf{b}_{2}, \ldots, \mathbf{a}+\mathbf{b}_{n-d}, \ldots, \mathbf{b}_{n}, \mathbf{f}^{\mathrm{T}}\right]^{\mathrm{T}} \in \mathbb{R}^{n^{2}+n}, \\
\boldsymbol{\theta}_{n} & :=\left[g_{1}, g_{2}, \ldots, g_{n_{g}}\right]^{\mathrm{T}} \in \mathbb{R}^{n_{g}} .
\end{aligned}
$$

When $d \geq n$, define the information vector $\varphi(k)$ and the parameter vector $\theta$ :

$$
\begin{aligned}
\boldsymbol{\varphi}(k) & :=\left[\boldsymbol{\varphi}_{1}^{\mathrm{T}}(k), \boldsymbol{\varphi}_{n}^{\mathrm{T}}(k)\right]^{\mathrm{T}}, \\
\boldsymbol{\varphi}_{1}(k) & :=\left[\begin{array}{c}
\mathbf{x}^{\mathrm{T}}(k-n), \mathbf{x}^{\mathrm{T}}(k-d-1), \mathbf{x}^{\mathrm{T}}(k-d-2), \ldots, \mathbf{x}^{\mathrm{T}}(k-d-n+1), \mathbf{x}^{\mathrm{T}}(k-d-n), u(k-1), u(k-2), \ldots, u(k-n)^{\mathrm{T}}
\end{array}\right]^{\mathrm{T}} \in \mathbb{R}^{n^{2}+2 n}, \\
\boldsymbol{\varphi}_{n}(k) & :=\left[v(k-1), v(k-2), \ldots, v\left(k-n_{g}\right)\right]^{\mathrm{T}} \in \mathbb{R}^{n_{g}}, \\
\boldsymbol{\theta} & :=\left[\boldsymbol{\theta}_{1}^{\mathrm{T}}, \boldsymbol{\theta}_{n}^{\mathrm{T}}\right]^{\mathrm{T}}, \\
\boldsymbol{\theta}_{1} & :=\left[\mathbf{a}, \mathbf{b}_{1}, \mathbf{b}_{2}, \ldots, \mathbf{b}_{n-1}, \mathbf{b}_{n}, \mathbf{f}^{\mathrm{T}}\right]^{\mathrm{T}} \in \mathbb{R}^{n^{2}+2 n}, \\
\boldsymbol{\theta}_{n} & :=\left[g_{1}, g_{2}, \ldots, g_{n_{g}}\right]^{\mathrm{T}} \in \mathbb{R}^{n_{g}} .
\end{aligned}
$$

From (2) and (7), we have

$$
y(k)=x_{1}(k)+w(k)=\varphi^{\mathrm{T}}(k) \boldsymbol{\theta}+w(k) .
$$

Multirate systems include dual-rate systems [30,31] and nonuniformly sampled systems $[32,33]$ as a special cases. In the dual-rate scheme, the observed output is sampled by the sampler and the sampling period is multiple of the input retention period. Assume that the sampling interval is $\tau$
( $\tau \geq 2$ is an integer), and thus, the measured input-output data are $\{u(k): k=0,1,2, \ldots\}$ at the fast rate and $\{y(k \tau): k=0,1,2, \ldots\}$ at the slow rate. Replacing $k$ in (10) with $k \tau$ gives

$$
y(k \tau)=x_{1}(k \tau)+w(k \tau)=\boldsymbol{\varphi}^{\mathrm{T}}(k \tau) \boldsymbol{\theta}+w(k \tau) .
$$

The identification model is important for parameter estimation, and many estimation algorithms have been 
proposed based on the identification models from observation data $[34,35]$ such as the gradient algorithms, the least squares algorithms, and the Newton algorithms [36].

Remark 2. This is the identification model of the dual-rate state-space system with $d$-step state-delay. The information vector $\varphi(k \tau)$ consists of the state vector $x(k \tau-i)$, the input $u(k \tau-i)$, and the correlated noise $w(k \tau-i)$, and the parameter vector $\theta$ consists of the parameters $a_{i}, b_{i}$, and $f_{i}$ of the state-space model in (1) and (2).

Remark 3. In what follows, a SG algorithm is derived for the state-space system with colored noise. Furthermore, a MISG algorithm is presented to reduce the computational burden and enhance the parameter estimation accuracy. A simulation example is provided to evaluate the estimation accuracy and the computational efficiency of the proposed algorithms.

\section{The Parameter and State Estimation Algorithm}

This section derives a multi-innovation stochastic gradient algorithm to estimate the parameter vector $\theta$ in (11) and uses the observer to estimate the state vector $x(k \tau)$ of the system.

4.1. The SG Algorithm. Defining and minimizing the cost function,

$$
J(\boldsymbol{\theta}):=\left[y(k \tau)-\boldsymbol{\varphi}^{\mathrm{T}}(k \tau) \boldsymbol{\theta}\right]^{2}
$$

and using the gradient search principle, we may obtain a stochastic gradient algorithm:

$$
\begin{aligned}
\widehat{\theta}(k \tau) & =\widehat{\theta}(k \tau-\tau)+\frac{\varphi(k \tau)}{r(k \tau)}\left[y(k \tau)-\varphi^{\mathrm{T}}(k \tau) \widehat{\theta}(k \tau-\tau)\right] \\
r(k \tau) & =r(k \tau-\tau)+\|\varphi(k \tau)\|^{2} \\
r(0) & =1
\end{aligned}
$$

where $1 / r(k \tau)$ is the step-size or convergence factor. The choice of $r(k \tau)$ guarantees that the parameter estimation error converges to zero. However, difficulties arise in that the information vector $\varphi(k \tau)$ contains the unknown state vector $x(k \tau-i)(i=1+d, 2+d, \ldots, n+d)$ and the SG algorithm in (13) and (14) cannot compute the estimate of $\theta$ in (11). The approach here is to replace the unknown $x(k \tau-i)$ in $\varphi(k \tau)$ with its $\hat{x}(k \tau-i)$. Based on the identification model in (11), we can obtain the following stochastic gradient parameter estimation algorithm for estimating $\theta$ :

$$
\begin{aligned}
\widehat{\theta}(k \tau) & =\widehat{\theta}(k \tau-\tau)+\frac{\widehat{\varphi}(k \tau)}{r(k \tau)}\left[y(k \tau)-\widehat{\varphi}^{\mathrm{T}}(k \tau) \widehat{\theta}(k \tau-\tau)\right], \\
r(k \tau) & =r(k \tau-\tau)+\|\widehat{\varphi}(k \tau)\|^{2} \\
r(0) & =1 .
\end{aligned}
$$

When $d \leq n-1$, we have

$$
\begin{aligned}
\widehat{\varphi}(k \tau) & =\left[\widehat{\varphi}_{1}^{\mathrm{T}}(k \tau), \widehat{\varphi}_{n}^{\mathrm{T}}(k \tau)\right]^{\mathrm{T}}, \\
\widehat{\varphi}_{1}(k \tau) & =\left[\begin{array}{c}
\hat{x}^{\mathrm{T}}(k \tau-d-1), \widehat{x}^{\mathrm{T}}(k \tau-d-2), \ldots, \widehat{x}^{\mathrm{T}}(k \tau-n), \ldots, \widehat{x}^{\mathrm{T}}(k \tau-n-d), \\
u(k \tau-1), u(k \tau-2), \ldots, u(k \tau-n)
\end{array}\right]^{\mathrm{T}}, \\
\widehat{\varphi}_{n}(k \tau) & =\left[\widehat{v}(k \tau-1), \widehat{v}(k \tau-2), \ldots, \widehat{v}\left(k \tau-n_{g}\right)\right]^{\mathrm{T}} \in \mathbb{R}^{n_{g}} .
\end{aligned}
$$

When $d \geq n$, we have

$$
\begin{aligned}
\widehat{\varphi}(k \tau) & =\left[\hat{\varphi}_{1}^{\mathrm{T}}(k \tau), \hat{\varphi}_{n}^{\mathrm{T}}(k \tau)\right]^{\mathrm{T}}, \\
\widehat{\varphi}_{1}(k \tau) & =\left[\begin{array}{c}
\hat{x}^{\mathrm{T}}(k \tau-n), \widehat{x}^{\mathrm{T}}(k \tau-d-1), \ldots, \widehat{x}^{\mathrm{T}}(k \tau-d-n+1), \widehat{x}^{\mathrm{T}}(k \tau-d-n), \\
u(k \tau-1), u(k \tau-2), \ldots, u(k \tau-n)
\end{array}\right]^{\mathrm{T}}, \\
\widehat{\varphi}_{n}(k \tau) & =\left[\widehat{v}(k \tau-1), \widehat{v}(k \tau-2), \ldots, \hat{v}\left(k \tau-n_{g}\right)\right]^{\mathrm{T}} \in \mathbb{R}^{n_{g}} .
\end{aligned}
$$

4.2. The MISG Algorithm. In order to improve the accuracy of the SG algorithm, we extend the SG algorithm and derive a multi-innovation stochastic gradient algorithm by expanding the innovation length.

Define an innovation vector:

$$
E(p, k \tau)=\left[\begin{array}{c}
e(k \tau) \\
e(k \tau-1) \\
\vdots \\
e(k \tau-p+1)
\end{array}\right] \in \mathbb{R}^{p}
$$


where the positive integer $p$ represents the innovation length, and

$$
e(k \tau-i)=y(k \tau-i)-\widehat{\varphi}^{\mathrm{T}}(k \tau-i) \hat{\theta}(k \tau-i-\tau) .
$$

In general, one may think that the estimate $\widehat{\theta}(k \tau-\tau)$ is closer to $\theta$ than $\widehat{\theta}(k \tau-\tau)$ at time $k \tau-i(i=2,3,4$, $\ldots, p-1)$. Thus, the innovation vector is taken more reasonably to be

$$
E(p, k \tau)=\left[\begin{array}{c}
y(k \tau)-\widehat{\varphi}^{\mathrm{T}}(k \tau) \hat{\theta}(k \tau-\tau) \\
y(k \tau-1)-\widehat{\varphi}^{\mathrm{T}}(k \tau-1) \hat{\theta}(k \tau-\tau) \\
\vdots \\
y(k \tau-p+1)-\widehat{\varphi}^{\mathrm{T}}(k \tau-p+1) \hat{\theta}(k \tau-\tau)
\end{array}\right] \in \mathbb{R}^{p} .
$$

Defining the information matrix $\widehat{\Phi}(p, k \tau)$ and stacking output vector $Y(p, k \tau)$ as

$$
\begin{aligned}
\widehat{\Phi}(p, k \tau):= & {[\widehat{\varphi}(k \tau), \widehat{\varphi}(k \tau-1), \ldots, \widehat{\varphi}(k \tau-p+1)] } \\
& \in \mathbb{R}^{\left(n^{2}+n+n_{g}\right) \times p}, \\
Y(p, k \tau):= & {[y(k \tau), y(k \tau-1), \ldots, y(k \tau-p+1)]^{\mathrm{T}} } \\
& \in \mathbb{R}^{p},
\end{aligned}
$$

the innovation vector $E(p, k \tau)$ can be equivalently expressed as

$$
E(p, k \tau)=Y(p, k \tau)-\widehat{\Phi}^{\mathrm{T}}(p, k \tau) \hat{\theta}(k \tau-\tau) .
$$

Furthermore, we can obtain the following multi-innovation stochastic gradient algorithm with the innovation length $p$ :

$$
\begin{aligned}
\widehat{\theta}(k \tau) & =\widehat{\theta}(k \tau-\tau)+\frac{\widehat{\Phi}(p, k \tau)}{r(k \tau)} E(p, k \tau), \\
E(p, k \tau) & =Y(p, k \tau)-\widehat{\Phi}^{\mathrm{T}}(p, k \tau) \widehat{\theta}(k \tau-\tau), \\
r(k \tau) & =r(k \tau-\tau)+\|\widehat{\varphi}(p, k \tau)\|^{2}, \\
r(0) & =1, \\
Y(p, k \tau) & =[y(k \tau), y(k \tau-1), \ldots, y(k \tau-p+1)]^{T}, \\
\widehat{\Phi}(p, k \tau) & =[\hat{\varphi}(k \tau), \widehat{\varphi}(k \tau-1), \ldots, \widehat{\varphi}(k \tau-p+1)] .
\end{aligned}
$$

When $d \leq n-1$, we have

$$
\begin{aligned}
\widehat{\varphi}(k \tau) & =\left[\widehat{\varphi}_{1}^{\mathrm{T}}(k \tau), \hat{\varphi}_{n}^{\mathrm{T}}(k \tau)\right]^{\mathrm{T}}, \\
\widehat{\varphi}_{n}(k \tau) & =\left[\begin{array}{c}
\hat{x}^{\mathrm{T}}(k \tau-d-1), \widehat{x}^{\mathrm{T}}(k \tau-d-2), \ldots, \widehat{x}^{\mathrm{T}}(k \tau-n), \ldots, \widehat{x}^{\mathrm{T}}(k \tau-n-d) \\
u(k \tau-1), u(k \tau-2), \ldots, u(k \tau-n)
\end{array}\right]^{\mathrm{T}}, \\
\widehat{\varphi}_{n}(k \tau) & =\left[\widehat{v}(k \tau-1), \widehat{v}(k \tau-2), \ldots, \widehat{v}\left(k \tau-n_{g}\right)\right]^{\mathrm{T}} \in \mathbb{R}^{n_{g}} .
\end{aligned}
$$

When $d \geq n$, we have

$$
\begin{aligned}
\widehat{\varphi}(k \tau) & =\left[\widehat{\varphi}_{1}^{\mathrm{T}}(k \tau), \widehat{\varphi}_{n}^{\mathrm{T}}(k \tau)\right]^{\mathrm{T}}, \\
\widehat{\varphi}_{1}(k \tau) & =\left[\begin{array}{c}
\hat{x}^{\mathrm{T}}(k \tau-n), \widehat{x}^{\mathrm{T}}(k \tau-d-1), \ldots, \widehat{x}^{\mathrm{T}}(k \tau-d-n+1), \\
\widehat{x}^{\mathrm{T}}(k \tau-d-n), u(k \tau-1), u(k \tau-2), \ldots, u(k \tau-n)
\end{array}\right]^{\mathrm{T}}, \\
\widehat{\varphi}_{n}(k \tau) & =\left[\widehat{v}(k \tau-1), \widehat{v}(k \tau-2), \ldots, \widehat{v}\left(k \tau-n_{g}\right)\right]^{\mathrm{T}} \in \mathbb{R}^{n_{g}} .
\end{aligned}
$$

When the innovation length $p=1$, the MISG algorithm degrades to the SG algorithm.

Theorem 1. For the system in (11) and the MISG algorithm in (24)-(34), assume that the system noise $v(k \tau)$ is random noise with zero mean and variance $\sigma^{2}$ that is uncorrelated with the input $u(k \tau)$, and the mean square is bounded, that is, (A1) $E[v(k \tau)]=0, E\left[\|v(k \tau)\|^{2}\right]=\sigma^{2}(k \tau) \leq \sigma^{2}$; the existence of constant $0<\alpha \leq \beta<\infty$ and integer $p \geq n$ make the following condition: (A2) $\alpha I \leq(1 / p) \sum_{i=1}^{p} \varphi(k \tau-i+1) \varphi^{T}(k \tau-i+1) \leq$ $\beta I$, a.s., $k \tau \geq p$. Then, the mean square error of the parameter estimation error given by MISG algorithm is bounded, that is, $\limsup _{k \tau \rightarrow \infty} E\left[\|\hat{\theta}(k \tau)-\theta\|^{2}\right] \leq 4 \beta^{3} \sigma^{2} / \alpha^{4}$.

4.3. The State Estimation Algorithm. Using the parameter estimation vector $\widehat{\theta}(k \tau)$ to form the system matrices/vectors $\widehat{A}(k \tau), \widehat{B}(k \tau)$, and $\hat{f}(k \tau)$ and based on the canonical state- 
space model in (1)-(2), we can use the following observer to estimate the state vector $x(k \tau)$ :

$$
\widehat{x}(k \tau+\tau)=\widehat{A}(k \tau) \widehat{x}(k \tau)+\widehat{B}(k \tau) \widehat{x}(k \tau-d \tau)+\widehat{f}(k \tau) u(k \tau),
$$

$$
\begin{aligned}
& \widehat{A}(k \tau)=\left[\begin{array}{cccc}
0 & 1 & \cdots & 0 \\
0 & 0 & \ddots & \vdots \\
\vdots & \vdots & \ddots & 0 \\
0 & 0 & 0 & 1 \\
\widehat{a}_{n}(k \tau) & \widehat{a}_{n-1}(k \tau) & \cdots & \widehat{a}_{1}(k \tau)
\end{array}\right] \\
& \widehat{B}(k \tau)=\left[\begin{array}{c}
\widehat{b}_{1}(k \tau) \\
\widehat{b}_{2}(k \tau) \\
\vdots \\
\widehat{b}_{n}(k \tau)
\end{array}\right] \text {, } \\
& \hat{f}(k \tau)=\left[\begin{array}{c}
\widehat{f}_{1}(k \tau) \\
\widehat{f}_{2}(k \tau) \\
\vdots \\
\widehat{f}_{n}(k \tau)
\end{array}\right] \text {, } \\
& \widehat{\theta}(k \tau)=\left[\widehat{b}_{1}(k \tau), \widehat{b}_{2}(k \tau), \ldots, \widehat{a}(k \tau)+\widehat{b}_{n-d}(k \tau),\right. \\
& \left.\ldots, \widehat{b}_{n}(k \tau), \hat{f}^{T}(k \tau)\right]^{T}, \quad d \leq n-1, \\
& \widehat{\theta}(k \tau)=\left[\widehat{a}(k \tau), \widehat{b}_{1}(k \tau), \widehat{b}_{2}(k \tau), \ldots, \widehat{b}_{n-1}(k \tau),\right. \\
& \left.\widehat{b}_{n}(k \tau), \hat{f}^{T}(k \tau)\right]^{T}, \quad d \geq n .
\end{aligned}
$$

The proposed algorithms in this paper can combine some mathematical tools [37] and other identification methods [38-40] to investigate the parameter identification methods of other linear and nonlinear systems [41-49] and can be applied to other literature studies [50-53] such as engineering application systems.

The steps of computing the parameter estimate $\widehat{\theta}(k \tau)$ in (23)-(28) and the state estimate $\hat{x}(k \tau+\tau)$ in (34)-(39) are listed in the following:

(1) Let $k=1$ and set the initial values $\hat{\theta}(0)=1_{n^{2}+2 n} / p_{0}$, $r(0)=1$, and $p_{0}=10^{6}$.

(2) Collect the input-output data $u(k \tau)$ and $y(k \tau)$ and form $\widehat{\varphi}(k \tau)$ by (29) or (32), $\widehat{\Phi}(p, k \tau)$ by $(28)$, and $Y(p, k \tau)$ by $(27)$, respectively.

(3) Compute $E(p, k \tau)$ by (25) and $r(k \tau)$ by (26).

(4) Update the parameter estimation vector $\hat{\theta}(k \tau)$ by (24).

(5) Read $\widehat{a}_{i}(k \tau), \hat{b}_{i}(k \tau)$, and $\widehat{f}(k \tau)$ from $\hat{\theta}(k \tau)$ according to the definition of $\widehat{\theta}(k \tau)$.

(6) Form $\widehat{A}(k \tau)$ and $\widehat{B}(k \tau)$ by (36) and (37).

(7) Compute the state estimation vector $\hat{x}(k \tau+\tau)$ by (35).
(8) Increase $k$ by 1 and go to step 2, and continue the recursive calculation.

Remark 4. The flop number is used to measure the calculation efficiency (calculation amount) of a complex algorithm. The total number of four floating-point operations required by an algorithm is defined as its calculation amount. Based on this, the calculation efficiency of the algorithm is evaluated as a benchmark, and an efficient and economical algorithm is sought. The calculation method is necessary to analyze the performance of the proposed algorithm.

The computational efficiency of the MISG and the SG algorithms is shown in Tables 1-3. Total floating-point operation (flop) numbers of the MISG and the SG algorithms are $N_{1}=\left(n^{2}+n+n_{g}\right)(4 p+3)$ and $N_{2}=7\left(n^{2}+\right.$ $\left.n+n_{g}\right)$, respectively. The difference between the MISG algorithm and the SG algorithm is

$$
\begin{aligned}
N_{1}-N_{2} & =\left(n^{2}+n+n_{g}\right)(4 p+3)-7\left(n^{2}+n+n_{g}\right) \\
& =(4 p-4)\left(n^{2}+n+n_{g}\right)>0 .
\end{aligned}
$$

Thus, the SG algorithm has smaller computational efforts than the MISG algorithm.

\section{Example}

Consider the following dual-rate time-delay system with $\tau=2$ :

$$
\begin{aligned}
x(t+1)= & {\left[\begin{array}{cc}
0 & 1 \\
-0.01 & -0.22
\end{array}\right] x(t)+\left[\begin{array}{rr}
0.19 & -0.08 \\
0.16 & -0.12
\end{array}\right] x(t-2) } \\
& +\left[\begin{array}{c}
0.8 \\
-0.8
\end{array}\right] u(t) \\
y(t)= & {[1,0] x(t)+v(t) . }
\end{aligned}
$$

The parameter vector to be identified is

$$
\begin{aligned}
\theta & =\left[a_{2}, a_{1}, b_{11}, b_{12}, b_{21}, b_{22}, f_{1}, f_{2}\right]^{\mathrm{T}} \\
& =[-0.01,-0.22,0.19,-0.08,0.16,-0.12,0.8,-0.8]^{\mathrm{T}} .
\end{aligned}
$$

In simulation, the input $\{u(t)\}$ is taken as an uncorrelated persistent excitation signal sequence with zero mean and unit variance, and $\{v(t)\}$ as a white noise sequence with zero mean and variances $\sigma^{2}=0.30^{2}$ and $\sigma^{2}=1.00^{2}$. The parameter estimation based MISG algorithm in (24)-(34) to estimate the parameter vector $\theta$ and the state estimation algorithm in (35)-(39) to estimate the state vector $x(t)$ of this example system are applied. The parameter estimates and their estimation errors are shown in Tables 4 and 5 and the parameter estimation errors $\delta$ versus $t$ are shown in Figures 1 and 2 with $p=1,2,5$, respectively, and the state estimates $\hat{x}_{1}(t)$ and $\widehat{x}_{2}(t)$ versus $t$ are shown in Figures 3 and 4 .

From Tables 4 and 5 and Figures 1-4, we can draw the following conclusions: 
TABLE 1: The computational burden of the MISG algorithm.

\begin{tabular}{lcr}
\hline Computational sequences & Number of multiplications & Number of additions \\
\hline$\widehat{\theta}(k \tau)=\widehat{\theta}(k \tau-\tau)+(\widehat{\Phi}(p, k \tau) / r(k \tau)) E(p, k \tau)$ & $\left(n^{2}+n+n_{g}\right)(p+1)$ & $\left(n^{2}+n+n_{g}\right) p$ \\
$E(p, k \tau)=Y(p, k \tau)-\widehat{\Phi}^{\mathrm{T}}(p, k \tau) \hat{\theta}(k \tau-\tau)$ & $\left(n^{2}+n+n_{g}\right) p$ & $\left(n^{2}+n+n_{g}\right) p$ \\
$r(k \tau)=r(k \tau-\tau)+\|\hat{\varphi}(p, k \tau)\|^{2}$ & $n^{2}+n+n_{g}$ & $n^{2}+n+n_{g}$ \\
\hline Sum & $2\left(n^{2}+n+n\right)(p+1)$ & $\left(n^{2}+n+n_{g}\right)(2 p+1)$ \\
\hline Total flops & & $\left(n^{2}+n+n\right)(4 p+3)$ \\
\hline
\end{tabular}

TABLe 2: The computational efficiency of the SG algorithm.

\begin{tabular}{lcc}
\hline Computational sequences & Number of multiplications & Number of additions \\
\hline$\widehat{\theta}(k \tau)=\widehat{\theta}(k \tau-\tau)+(\widehat{\varphi}(k \tau) / r(k \tau))\left[y(k \tau)-\widehat{\varphi}^{\mathrm{T}}(k \tau) \hat{\theta}(k \tau-\tau)\right]$ & $3\left(n^{2}+n+n_{g}\right)$ & $2\left(n^{2}+n+n_{g}\right)$ \\
$r(k \tau)=r(k \tau-\tau)+\|\widehat{\varphi}(k \tau)\|^{2}$ & $n^{2}+n+n$ & $n^{2}+n+n$ \\
\hline Sum & $4\left(n^{2}+n+n_{q}\right)$ & $3\left(n^{2}+n+n_{q}\right)$ \\
\hline Total flops & & $7\left(n^{2}+n+n\right)$ \\
\hline
\end{tabular}

TABLE 3: Comparison of the computational efficiency of the MISG and the SG algorithms.

\begin{tabular}{lccc}
\hline Algorithms & Number of multiplications & Number of additions & Total flops \\
\hline MISG & $2\left(n^{2}+n+n_{g}\right)(p+1)$ & $\left(n^{2}+n+n_{g}\right)(2 p+1)$ & $\left(n^{2}+n+n_{g}\right)(4 p+3)$ \\
\hline SG & $4\left(n^{2}+n+n_{g}\right)$ & $3\left(n^{2}+n+n_{g}\right)$ & $7\left(n^{2}+n+n_{g}\right)$ \\
\hline
\end{tabular}

TABLE 4: The parameter estimates and errors with $\sigma^{2}=0.30^{2}$.

\begin{tabular}{|c|c|c|c|c|c|c|c|c|c|c|}
\hline Algorithms & $t$ & $a_{2}$ & $a_{1}$ & $b_{11}$ & $b_{12}$ & $b_{21}$ & $b_{22}$ & $f_{1}$ & $f_{2}$ & $\delta(\%)$ \\
\hline \multirow{6}{*}{ SG (MISG, $p=1)$} & 100 & -0.00912 & -0.15036 & 0.04307 & -0.06630 & 0.02505 & -0.01004 & 0.49833 & -0.38674 & 47.52683 \\
\hline & 200 & -0.00071 & -0.15897 & 0.03430 & -0.06226 & 0.03583 & -0.01874 & 0.51905 & -0.41671 & 44.53744 \\
\hline & 500 & -0.01158 & -0.16218 & 0.04174 & -0.07458 & 0.04926 & -0.02578 & 0.55028 & -0.45958 & 39.90966 \\
\hline & 1000 & -0.01300 & -0.16742 & 0.04407 & -0.07779 & 0.05693 & -0.02935 & 0.57120 & -0.48809 & 36.94100 \\
\hline & 2000 & -0.01608 & -0.17243 & 0.04797 & -0.08160 & 0.06551 & -0.03294 & 0.58943 & -0.51204 & 34.32511 \\
\hline & 3000 & -0.01644 & -0.17616 & 0.04817 & -0.08312 & 0.07199 & -0.03621 & 0.59949 & -0.52557 & 32.86166 \\
\hline \multirow{6}{*}{ MISG, $p=2$} & 100 & 0.00359 & -0.10678 & 0.07097 & -0.11681 & 0.07671 & -0.04656 & 0.66219 & -0.54089 & 29.99962 \\
\hline & 200 & 0.01599 & -0.12109 & 0.06185 & -0.11177 & 0.09003 & -0.05590 & 0.68106 & -0.57759 & 26.6 \\
\hline & 500 & 0.00850 & -0.13309 & 0.07485 & -0.12515 & 0.10632 & -0.06220 & 0.70645 & -0.62350 & 22.16532 \\
\hline & 1000 & 0.01018 & -0.14260 & 0.07880 & -0.12629 & 0.11409 & -0.06384 & 0.72115 & -0.65182 & 19.62474 \\
\hline & 2000 & 0.00966 & -0.15129 & 0.08537 & -0.12916 & 0.12141 & -0.06584 & 0.73406 & -0.67410 & 17.47593 \\
\hline & 3000 & 0.01029 & -0.15688 & 0.08764 & -0.13042 & 0.12688 & -0.06787 & 0.74038 & -0.68567 & 16.37969 \\
\hline \multirow{6}{*}{ MISG, $p=5$} & 100 & -0.01604 & -0.16063 & 0.16648 & -0.04942 & 0.12274 & -0.11018 & 0.77804 & -0.72833 & 9.28037 \\
\hline & 200 & -0.00941 & -0.17261 & 0.16361 & -0.05183 & 0.13136 & -0.11273 & 0.78161 & -0.75681 & 6.94835 \\
\hline & 500 & -0.01370 & -0.18867 & 0.17725 & -0.06884 & 0.14888 & -0.11953 & 0.79311 & -0.77446 & 3.86359 \\
\hline & 1000 & -0.00956 & -0.19552 & 0.17681 & -0.06720 & 0.15297 & -0.11685 & 0.79300 & -0.78499 & 3.00103 \\
\hline & 2000 & -0.00865 & -0.20173 & 0.18160 & -0.07054 & 0.15557 & -0.11647 & 0.79656 & -0.79077 & 2.10426 \\
\hline & 3000 & -0.00843 & -0.20539 & 0.18430 & -0.07311 & 0.15762 & -0.11675 & 0.79793 & -0.79282 & 1.61476 \\
\hline True values & & -0.01000 & -0.22000 & 0.19000 & -0.08000 & 0.16000 & -0.12000 & 0.80000 & -0.80000 & \\
\hline
\end{tabular}

TABLE 5: The parameter estimates and errors with $\sigma^{2}=1.00^{2}$.

\begin{tabular}{lcccccccccc}
\hline Algorithms & $t$ & $a_{2}$ & $a_{1}$ & $b_{11}$ & $b_{12}$ & $b_{21}$ & $b_{22}$ & $f_{1}$ & $f_{2}$ & $\delta(\%)$ \\
\hline & 100 & -0.01550 & -0.15470 & 0.04028 & -0.06265 & 0.02094 & -0.01630 & 0.52050 & -0.34565 & 49.20737 \\
& 200 & -0.00846 & -0.16114 & 0.03142 & -0.05900 & 0.02910 & -0.02302 & 0.53817 & -0.38165 & 46.04580 \\
SG (MISG, $p=1)$ & 500 & -0.02283 & -0.16425 & 0.04190 & -0.07513 & 0.04531 & -0.03215 & 0.57022 & -0.42744 & 41.04400 \\
& 1000 & -0.02246 & -0.16947 & 0.04135 & -0.07521 & 0.05323 & -0.03429 & 0.58828 & -0.45950 & 38.05525 \\
& 2000 & -0.02481 & -0.17522 & 0.04532 & -0.07876 & 0.06177 & -0.03769 & 0.60570 & -0.48594 & 35.31488 \\
& 3000 & -0.02492 & -0.17967 & 0.04580 & -0.08054 & 0.06861 & -0.04099 & 0.61533 & -0.50079 & 33.76525 \\
\hline
\end{tabular}


TABLE 5: Continued.

\begin{tabular}{lcccccccccc}
\hline Algorithms & $t$ & $a_{2}$ & $a_{1}$ & $b_{11}$ & $b_{12}$ & $b_{21}$ & $b_{22}$ & $f_{1}$ & $f_{2}$ & $\delta(\%)$ \\
\hline & 100 & -0.00070 & -0.09901 & 0.08115 & -0.11549 & 0.07387 & -0.05378 & 0.68241 & -0.51521 & 30.88244 \\
& 200 & 0.01133 & -0.11098 & 0.06966 & -0.10975 & 0.08403 & -0.06043 & 0.69602 & -0.56265 & 27.17174 \\
MISG, $p=2$ & 500 & 0.00040 & -0.12474 & 0.08572 & -0.12822 & 0.10703 & -0.07177 & 0.72361 & -0.60977 & 22.19946 \\
& 1000 & 0.00665 & -0.13482 & 0.08321 & -0.12254 & 0.11560 & -0.07075 & 0.73290 & -0.64205 & 19.67043 \\
& 2000 & 0.00802 & -0.14527 & 0.08971 & -0.12475 & 0.12278 & -0.07232 & 0.74507 & -0.66653 & 17.34685 \\
& 3000 & 0.00917 & -0.15233 & 0.09273 & -0.12666 & 0.12873 & -0.07427 & 0.75100 & -0.67898 & 16.12797 \\
\hline & 100 & -0.02959 & -0.13914 & 0.19948 & -0.01531 & 0.08421 & -0.15341 & 0.76481 & -0.73279 & 12.99066 \\
& 200 & -0.01861 & -0.15341 & 0.18733 & -0.01475 & 0.09415 & -0.14951 & 0.76310 & -0.78040 & 10.55685 \\
MISG, $p=5$ & 500 & -0.02916 & -0.17950 & 0.20785 & -0.04599 & 0.13403 & -0.16618 & 0.79390 & -0.78371 & 6.83573 \\
& 1000 & -0.01718 & -0.18774 & 0.19516 & -0.03222 & 0.14082 & -0.15378 & 0.78631 & -0.79542 & 6.02358 \\
& 2000 & -0.01503 & -0.19804 & 0.20265 & -0.03826 & 0.14446 & -0.14983 & 0.79412 & -0.79944 & 5.03453 \\
& 3000 & -0.01509 & -0.20501 & 0.20834 & -0.04462 & 0.14803 & -0.14864 & 0.79741 & -0.80005 & 4.46176 \\
\hline True values & & -0.01000 & -0.22000 & 0.19000 & -0.08000 & 0.16000 & -0.12000 & 0.80000 & -0.80000 & \\
\hline
\end{tabular}

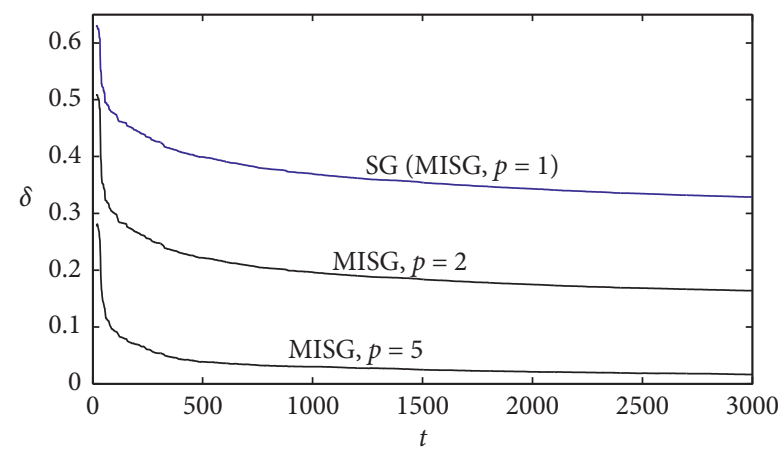

Figure 1: The parameter estimation errors $\delta$ versus $t$ with $\sigma^{2}=0.30^{2}$.

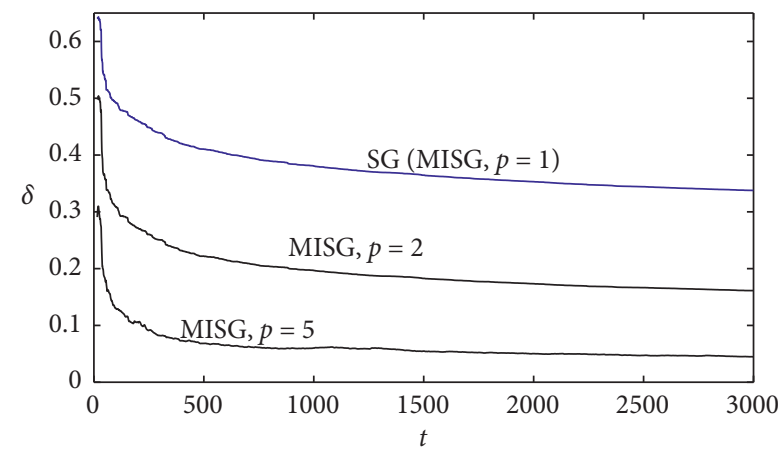

Figure 2: The parameter estimation errors $\delta$ versus $t$ with $\sigma^{2}=1.00^{2}$.

(1) The parameter estimates converge fast to their true values for large $p$, see Tables 4 and 5

(2) The MISG algorithm with $p \geq 2$ has higher accuracy than the SG algorithm, see Figures 1 and 2

(3) The parameter estimation errors given by the MISG algorithm become smaller with the data length $t$ and the innovation length $p$ increasing, see Tables 4 and 5 and Figures 1 and 2

(4) The state estimates are close to their true values with $t$ increasing, see Figures 3 and 4

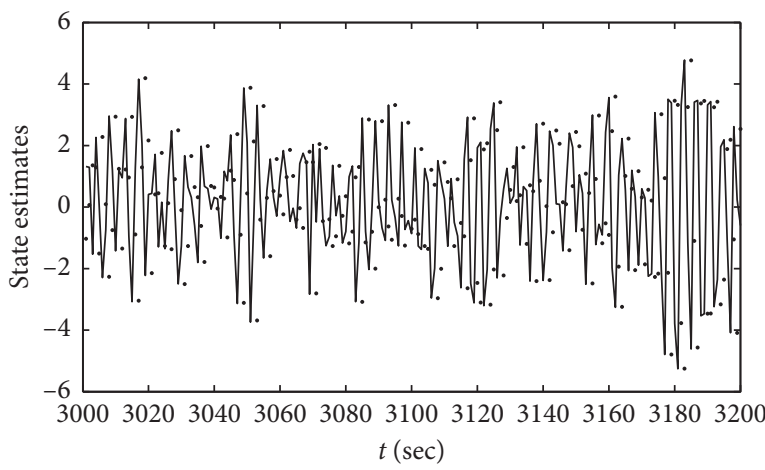

FIgURE 3: The state and state estimate $\widehat{x}_{1}(t)$ versus $t$; solid line: the true $x_{1}(t)$; dots: the estimated $\widehat{x}_{1}(t)$.

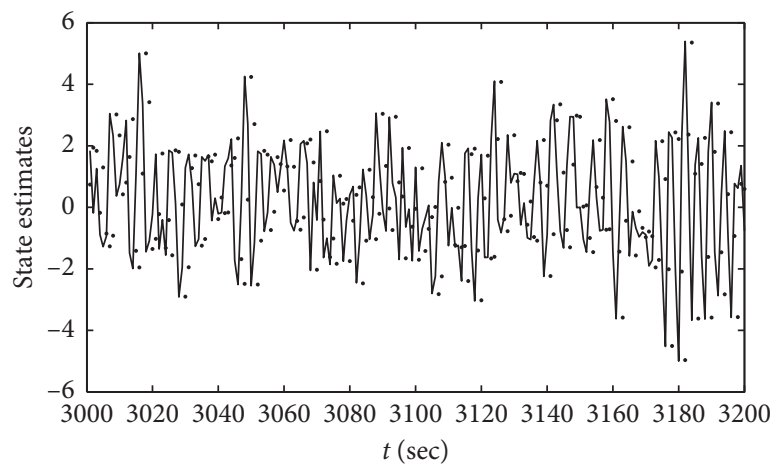

Figure 4: The state and state estimate $\widehat{x}_{2}(t)$ versus $t$; solid line: the true $x_{2}(t)$; dots: the estimated $\hat{x}_{2}(t)$.

\section{Conclusions}

This study has taken up a category of state-space models with state time delay as the research background and accordingly developed two folds of solutions for the model identification (parameter and state estimation in specific). The theoretical analysis has proved that the estimates converge to the real value under the condition of continuous excitation in modelling. The algorithms used in this paper can be applied to hybrid switched impulsive power networks and uncertain 
chaotic nonlinear systems with time delay [54-57] and can be applied to other literature studies [58-64]. The simulation case study has demonstrated that the proposed algorithms/ procedures are effective and efficient in design and implementation.

\section{Data Availability}

No data were used to support this study.

\section{Conflicts of Interest}

The authors declare that there are no conflicts of interest regarding the publication of this paper.

\section{Acknowledgments}

This work was supported by the National Natural Science Foundation of China (nos. 61903050 and 61673277), the Natural Science Foundation of Jiangsu Province (no. BK20181033), the Natural Science Fundamental Research Project of Colleges and Universities in Jiangsu Province (no. 18KJB120001), the Science and Technology Development Plan Project of Changshu under grant no. CR201711, and Jiangsu Planned Projects for Postdoctoral Research Funds.

\section{References}

[1] F. Ding, G. Liu, and X. P. Liu, "Parameter estimation with scarce measurements," Automatica, vol. 47, no. 8, pp. 16461655, 2011.

[2] D. Wang, S. Zhang, M. Gan, and J. Qiu, "A novel EM identification method for Hammerstein systems with missing output data," IEEE Transactions on Industrial Informatics, vol. 16, no. 4, pp. 2500-2508, 2020.

[3] M. Li, X. Liu, and F. Ding, "The filtering-based maximum likelihood iterative estimation algorithms for a special class of nonlinear systems with autoregressive moving average noise using the hierarchical identification principle," International Journal of Adaptive Control and Signal Processing, vol. 33, no. 7, pp. 1189-1211, 2019.

[4] F. Ding, "Hierarchical multi-innovation stochastic gradient algorithm for Hammerstein nonlinear system modeling," Applied Mathematical Modelling, vol. 37, no. 4, pp. 16941704, 2013.

[5] F. Ding, "State filtering and parameter estimation for state space systems with scarce measurements," Signal Processing, vol. 104, pp. 369-380, 2014.

[6] F. Ding, "Combined state and least squares parameter estimation algorithms for dynamic systems," Applied Mathematical Modelling, vol. 38, no. 1, pp. 403-412, 2014.

[7] J. Chen, Y. Zhang, Q. Zhu, and Y. Liu, “Aitken based modified Kalman filtering stochastic gradient algorithm for dual-rate nonlinear models," Journal of the Franklin Institute, vol. 356, no. 8, pp. 4732-4746, 2019.

[8] J. Ding, J. Chen, J. Lin, and L. Wan, "Particle filtering based parameter estimation for systems with output-error type model structures," Journal of the Franklin Institute, vol. 356, no. 10, pp. 5521-5540, 2019.

[9] J. Ding, J. Chen, J. Lin, and G. Jiang, "Particle filtering-based recursive identification for controlled auto-regressive systems with quantised output," IET Control Theory \& Applications, vol. 13, no. 14, pp. 2181-2187, 2019.
[10] Y. Gu, J. Liu, X. Li, Y. Chou, and Y. Ji, "State space model identification of multirate processes with time-delay using the expectation maximization," Journal of the Franklin Institute, vol. 356, no. 3, pp. 1623-1639, 2019.

[11] Y. Gu, Y. Chou, J. Liu, and Y. Ji, "Moving horizon estimation for multirate systems with time-varying time-delays," Journal of the Franklin Institute, vol. 356, no. 4, pp. 2325-2345, 2019.

[12] M. Li and X. Liu, "The least squares based iterative algorithms for parameter estimation of a bilinear system with autoregressive noise using the data filtering technique," Signal Processing, vol. 147, pp. 23-34, 2018.

[13] X. Zhang and F. Ding, "Hierarchical parameter and state estimation for bilinear systems," International Journal of Systems Science, vol. 51, no. 2, pp. 275-290, 2020.

[14] M. Li and X. Liu, "Maximum likelihood least squares based iterative estimation for a class of bilinear systems using the data filtering technique," International Journal of Control, Automation and Systems, vol. 18, no. 6, pp. 1581-1592, 2020.

[15] J. Ding, Z. Cao, J. Chen, and G. Jiang, "Weighted parameter estimation for hammerstein nonlinear ARX systems," Circuits, Systems, and Signal Processing, vol. 39, no. 4, pp. 2178-2192, 2020.

[16] F. Ding, X. P. Liu, and G. Liu, "Identification methods for Hammerstein nonlinear systems," Digital Signal Processing, vol. 21, no. 2, pp. 215-238, 2011.

[17] M. Gan, G.-Y. Chen, L. Chen, and C. L. P. Chen, "Term selection for a class of separable nonlinear models," IEEE Transactions on Neural Networks and Learning Systems, vol. 31, no. 2, pp. 445-451, 2020.

[18] F. Ding, L. Xu, D. D. Meng et al., "Gradient estimation algorithms for the parameter identification of bilinear systems using the auxiliary model," Journal of Computational and Applied Mathematics, vol. 369, 2020.

[19] D. Wang, L. Li, Y. Ji, and Y. Yan, "Model recovery for Hammerstein systems using the auxiliary model based orthogonal matching pursuit method," Applied Mathematical Modelling, vol. 54, pp. 537-550, 2018.

[20] G. Y. Chen, M. Gan, C. L. P. Chen, and H. X. Li, "Basis function matrix based flexible coefficient autoregressive models: a framework for time series and nonlinear system modeling," IEEE Transactions on Cybernetics, 2019, In press.

[21] F. Ding, L. Lv, J. Pan, X. Wan, and X.-B. Jin, "Two-stage gradient-based iterative estimation methods for controlled autoregressive systems using the measurement data," International Journal of Control, Automation and Systems, vol. 18, no. 4, pp. 886-896, 2020.

[22] J. Chen, J. Li, and Y. Liu, "Gradient iterative algorithm for dual-rate nonlinear systems based on a novel particle filter," Journal of the Franklin Institute, vol. 354, no. 11, pp. 44254437, 2017.

[23] J. Pan, X. Jiang, X. Wan, and W. Ding, "A filtering based multi-innovation extended stochastic gradient algorithm for multivariable control systems," International Journal of Control, Automation and Systems, vol. 15, no. 3, pp. 11891197, 2017.

[24] F. Ding, X. Zhang, and L. Xu, “The innovation algorithms for multivariable state-space models," International Journal of Adaptive Control and Signal Processing, vol. 33, no. 11, pp. 1601-1618, 2019.

[25] C. Y. Dong, S. F. Yang, H. J. Jia, and P. Wang, "Pade-based stability analysis for a modular multilevel converter considering the time delay in the digital control system," IEEE Transactions on Industrial Electronics, vol. 66, no. 7, pp. 5242-5253, 2019. 
[26] S. Z. Yong, M. Zhu, and E. Frazzoli, "Simultaneous input and state estimation for linear time-varying continuous-time stochastic systems," IEEE Transactions on Automatic Control, vol. 62, no. 5, pp. 2531-2538, 2017.

[27] J. Chen, B. Huang, F. Ding, and Y. Gu, "Variational Bayesian approach for ARX systems with missing observations and varying time-delays," Automatica, vol. 94, pp. 194-204, 2018.

[28] R. Sanz, P. García, and M. Krstic, "Observation and stabilization of LTV systems with time-varying measurement delay," Automatica, vol. 103, pp. 573-579, 2019.

[29] D. Li, C. Zhang, and H. Qin, "LDG method for reactiondiffusion dynamical systems with time delay," Applied Mathematics and Computation, vol. 217, no. 22, pp. 91739181, 2011.

[30] Y. Liu, F. Ding, and Y. Shi, "An efficient hierarchical identification method for general dual-rate sampled-data systems," Automatica, vol. 50, no. 3, pp. 962-970, 2014.

[31] J. Ding, F. Ding, X. P. Liu, and G. Liu, "Hierarchical least squares identification for linear SISO systems with dual-rate sampled-data," IEEE Transactions on Automatic Control, vol. 56, no. 11, pp. 2677-2683, 2011.

[32] F. Ding, G. Liu, and X. P. Liu, "Partially coupled stochastic gradient identification methods for non-uniformly sampled systems," IEEE Transactions on Automatic Control, vol. 55, no. 8, pp. 1976-1981, 2010.

[33] F. Ding, L. Qiu, and T. Chen, "Reconstruction of continuoustime systems from their non-uniformly sampled discrete-time systems," Automatica, vol. 45, no. 2, pp. 324-332, 2009.

[34] L. Xu, L. Chen, and W. Xiong, "Parameter estimation and controller design for dynamic systems from the step responses based on the Newton iteration," Nonlinear Dynamics, vol. 79, no. 3, pp. 2155-2163, 2015.

[35] L. $\mathrm{Xu}$, "The damping iterative parameter identification method for dynamical systems based on the sine signal measurement," Signal Processing, vol. 120, pp. 660-667, 2016.

[36] L. Xu, W. Xiong, A. Alsaedi, and T. Hayat, "Hierarchical parameter estimation for the frequency response based on the dynamical window data," International Journal of Control, Automation and Systems, vol. 16, no. 4, pp. 1756-1764, 2018.

[37] Y. Wang, G. Zhuang, and F. Chen, "A dynamic event-triggered Ho control for singular Markov jump systems with redundant channels," International Journal of Systems Science, vol. 51, no. 1, pp. 158-179, 2020.

[38] X. Zhang, F. Ding, L. Xu, and E. Yang, "Highly computationally efficient state filter based on the delta operator," International Journal of Adaptive Control and Signal Processing, vol. 33, no. 6, pp. 875-889, 2019.

[39] X. Zhang and F. Ding, "Adaptive parameter estimation for a general dynamical system with unknown states," International Journal of Robust and Nonlinear Control, vol. 30, no. 4, pp. 1351-1372, 2020.

[40] Y. Wang, G. Zhuang, X. Chen, Z. Wang, and F. Chen, "Dynamic event-based finite-time mixed $H \infty$ and passive asynchronous filtering for T-S fuzzy singular Markov jump systems with general transition rates," Nonlinear Analysis: Hybrid Systems, vol. 36, Article ID 100874, 2020.

[41] Y. Chang, G. Zhai, B. Fu, and L. Xiong, "Quadratic stabilization of switched uncertain linear systems: a convex combination approach," IEEE/CAA Journal of Automatica Sinica, vol. 6, no. 5, pp. 1116-1126, 2019.

[42] Y. Chang, C. Sun, and Y. Qiu, "Effective notch stress method for fatigue assessment of sheet alloy material and bi-material welded joints," Thin-Walled Structures, vol. 151, Article ID $106745,2020$.
[43] L. Geng and R. B. Xiao, "Control and backbone identification for the resilient recovery of a supply network utilizing outer synchronization,” Applied Sciences, vol. 10, no. 1, p. 313, 2020.

[44] L. He, H. Lin, Q. Zou, and D. Zhang, "Accurate measurement of pavement deflection velocity under dynamic loads," $A u$ tomation in Construction, vol. 83, pp. 149-162, 2017.

[45] L. Wang, B. Feng, Y. Wang, T. Wu, and H. Lin, "Bidirectional short-circuit current blocker for DC microgrid based on solidstate circuit breaker," Electronics, vol. 9, no. 2, p. 306, 2020.

[46] L. Wang, J. Guo, C. Xu, T. Wu, and H. Lin, "Hybrid model predictive control strategy of supercapacitor energy storage system based on double active bridge," Energies, vol. 12, no. 11, p. 2134, 2019.

[47] T. Wu, F. Ye, Y. Su, Y. Wang, and S. Riffat, "Coordinated control strategy of DC microgrid with hybrid energy storage system to smooth power output fluctuation," International Journal of Low-Carbon Technologies, vol. 15, no. 1, pp. 46-54, 2020.

[48] Y. Zhang, M. Huang, T. Wu, and F. Ji, "Reconfigurable equilibrium circuit with additional power supply," International Journal of Low-Carbon Technologies, vol. 15, no. 1, pp. 106-111, 2020.

[49] Y. Xing, J. Na, and R. Costa-Castello, "Real-time adaptive parameter estimation for a polymer electrolyte membrane fuel cell," IEEE Transactions on Industrial Informatics, vol. 15, no. 11, pp. 6048-6057, 2019.

[50] D. Wang, L. Mao, and F. Ding, "Recasted models-based hierarchical extended stochastic gradient method for MIMO nonlinear systems," IET Control Theory \& Applications, vol. 11, no. 4, pp. 476-485, 2017.

[51] G. Yang, Z. Chen, Y. Li, and Z. Su, "Rapid relocation method for mobile robot based on improved ORB-SLAM2 algorithm," Remote Sensing, vol. 11, no. 2, p. 149, 2019.

[52] Z. Su, Y. Li, and G. Yang, "Dietary composition perception algorithm using social robot audition for Mandarin Chinese," IEEE Access, vol. 8, pp. 8768-8782, 2020.

[53] L. Lv, S. Tang, and L. Zhang, "Parametric solutions to generalized periodic Sylvester bimatrix equations," Journal of the Franklin Institute, vol. 357, no. 6, pp. 3601-3621, 2020.

[54] D. Wang, Y. Yan, Y. Liu, and J. Ding, "Model recovery for Hammerstein systems using the hierarchical orthogonal matching pursuit method," Journal of Computational and Applied Mathematics, vol. 345, pp. 135-145, 2019.

[55] T. Zhang and F. Ma, "Improved rough K-Means clustering algorithm based on weighted distance measure with Gaussian function," International Journal of Computer Mathematics, vol. 94, no. 4, pp. 663-675, 2017.

[56] M. Gan, C. L. P. Chen, G.-Y. Chen, and L. Chen, "On some separated algorithms for separable nonlinear least squares problems," IEEE Transactions on Cybernetics, vol. 48, no. 10, pp. 2866-2874, 2018.

[57] G. Y. Chen, M. Gan, C. L. P. Chen, and H. X. Li, "A regularized variable projection algorithm for separable nonlinear least squares problems," IEEE Transactions on Automatic Control, vol. 64, no. 2, pp. 526-537, 2019.

[58] T. F. Zhang, F. M. Ma, D. Yue, C. Peng, and G. M. P. O’Hare, "Interval type-2 fuzzy local enhancement based rough K-means clustering considering imbalanced clusters," IEEE Transactions on Fuzzy Systems, 2019, In press.

[59] F. Ma, M. Ding, T. Zhang, and J. Cao, "Compressed binary discernibility matrix based incremental attribute reduction algorithm for group dynamic data," Neurocomputing, vol. 344, pp. 20-27, 2019. 
[60] J. Na, Y. S. Xing, and R. Costa-Castell, "Adaptive estimation of time-varying parameters with application to roto-magnet plant," IEEE Transactions on Systems, Man, and Cybernetics: Systems, 2018, In press.

[61] N. Zhao, Y.-C. Liang, D. Niyato, Y. Pei, M. Wu, and Y. Jiang, "Deep reinforcement learning for user association and resource allocation in heterogeneous cellular networks," IEEE Transactions on Wireless Communications, vol. 18, no. 11, pp. 5141-5152, 2019.

[62] Y. Zhang, X. Li, G. Zhao, B. Lu, and C. C. Cavalcante, "Signal reconstruction of compressed sensing based on alternating direction method of multipliers," Circuits, Systems, and Signal Processing, vol. 39, no. 1, pp. 307-323, 2020.

[63] L. Wang, H. Liu, L. V. Dai, and Y. W. Liu, "Novel method for identifying fault location of mixed lines," Energies, vol. 11, no. 6, Article ID 1529, 2018.

[64] H. Liu, Q. Zou, and Z. Zhang, "Energy disaggregation of appliances consumptions using ham approach," IEEE Access, vol. 7, pp. 185977-185990, 2019. 